\title{
A Novel Noninvasive Method for Quantitative Detection of Colonic Dysmotility Using Real-Time Ultrasonography
}

\author{
Kazuhisa Kishia ${ }^{a}$ Noriyuki Kaji ${ }^{a}$ b $\quad$ Yoshiharu Tsuru ${ }^{c}$ Masatoshi Hori ${ }^{a}$ \\ aDepartment of Veterinary Pharmacology, Graduate School of Agriculture and Life Sciences, The University of \\ Tokyo, Tokyo, Japan; ' Department of Pharmacology, School of Veterinary Medicine, Azabu University, Sagamihara, \\ Japan; 'Primetech Life Science Laboratory, PRIMETECH Corporation, Tokyo, Japan
}

\section{Keywords}

Colonic motility · Inflammatory bowel disease $\cdot$ Ulcerative colitis · Ultrasound

\begin{abstract}
Introduction: Colonic motility disorders are a frequent clinical problem caused by various drugs and diseases. However, the etiology of colonic dysmotility is often unclear due to the lack of in vivo methods, including rapid dynamic assessment. Objectives: The aim of this study was to establish a novel quantitative method to objectively assess colonic motility using ultrasonography. Methods: We applied echocardiographic speckle tracking-based strain imaging to analyze murine colonic motility. A trace line was placed on the boundary between the proximal wall of the colon and the inner cavity to analyze colonic wall displacement and strain rate. Locomotion activities of the colonic wall were used to quantify colonic motility via ultrasonography. Results: We found that ultrasonography can quantitatively detect a decrease in colonic motility induced by loperamide, an antidiarrheal drug. These quantitative data were consistent with the imaging findings of colonic peristalsis and colon transit time. Additionally, ultrasonography also revealed changes in
\end{abstract}

karger@karger.com www.karger.com/dig

Karger $\stackrel{\text { ' }}{5}$

GOPEN ACCESS
(C) 2020 The Author(s)

Published by S. Karger AG, Basel

This is an Open Access article licensed under the Creative Commons Attribution-NonCommercial-4.0 International License (CC BY-NC) (http://www.karger.com/Services/OpenAccessLicense), applicable to the online version of the article only. Usage and distribution for commercial purposes requires written permission. colonic motility over short intervals. Furthermore, we have shown that ultrasonography can quantitatively and noninvasively detect colonic dysmotility and hypervascularity of the colonic wall in colitis mice. Conclusions: These findings suggest that ultrasonography is a useful in vivo method for objectively monitoring changes in colonic motility caused by drugs and diseases.

(C) 2020 The Author(s) Published by S. Karger AG, Basel

\section{Introduction}

Gastrointestinal (GI) motility disorders are a major health problem in both humans and animals. Abnormal GI motility is caused by various diseases and drugs [1-3]. In fact, GI motility disorders, such as gastroparesis, irritable bowel syndrome, and inflammatory bowel disease (IBD), can cause clinical symptoms such as vomiting, abdominal pain, constipation, and diarrhea, greatly reducing the quality of life [4-6]. Disorders of GI motility frequently occur in daily life, and lower GI symptoms such as diarrhea and constipation are particularly serious clinical problems [2, 7]. However, the etiology of diarrhea and constipation of- 
ten remains unclear due to the lack of in vivo methods to objectively assess changes in colonic motility. Thus, the development of new in vivo methods is important for both basic medical research and clinical practice.

Basic medical research using rodents will help to understand changes in colonic motor function caused by diseases and drugs. Currently, a bead expulsion assay is used to assess colonic motility in rodents $[8,9]$. However, this method requires the insertion of beads into the intestinal lumen, and invasive manipulation can damage colon tissue. Thus, the measurements obtained by this method are highly variable both between and within individual animals [9]. This issue complicates the interpretation of data. Therefore, the development of more convenient and better analytical methods that enable the noninvasive assessment of colonic motor function is necessary in basic medical research.

Ultrasonography is often performed as a point-of-care examination in clinical practice and is a noninvasive diagnostic imaging method for assessing the condition of organs. Real-time ultrasonography provides a morphological assessment of the GI tract via imaging and helps detect abnormalities. The identifiable abnormalities include changes in intestinal blood flow using color Doppler ultrasound [10] as well as the loss of normal layer structure due to inflammation and tumors. Recently, GI motility measurements using ultrasonography have been performed to roughly estimate changes in gastric emptying [11-15]. On the other hand, there are few studies on the use of ultrasonography to assess changes in colonic motility because intestinal ultrasound is considered an in vivo method that is not highly quantitative. Intestinal ultrasound has methodological difficulties, and its assessment is influenced to some extent by the operator's subjectivity and skill $[16,17]$. If colonic motility can be quantified in addition to assessment by diagnostic imaging, a more objective assessment would be possible.

The aim of this study was to establish a novel in vivo method to objectively assess colonic motility using an ultrasonic imaging system for small animals. Here, we present advances in ultrasonography for the rapid quantification of colonic motility, allowing studies of the changes in colonic motor function and the effects of drugs and diseases. This research provides a new resource for the biomedical research community, including basic research and clinical applications. We validated the usefulness of ultrasonography for real-time monitoring of the colon using loperamide, an antidiarrheal drug, in a mouse model and a dextran sodium sulfate (DSS)-induced colitis mouse model.

\section{Methods}

Mice

We used 8- to 10 -week-old male C57BL/6J mice in this study. Mice were housed under controlled conditions $\left(22 \pm 2^{\circ} \mathrm{C}\right.$ on a $12-\mathrm{h}$ light/dark cycle) and were given free access to water and food. All procedures were approved by the Institutional Review Board of the University of Tokyo (approval code P18-131). The experimental procedures using mice complied with the Guide for Animal Use and Care published by the University of Tokyo.

\section{GI Hypomotility Mouse Model}

GI hypomotility model mice were prepared using loperamide (FUJIFILM Wako Pure Chemical, Tokyo, Japan), which is an antidiarrheal drug. C57BL/6J mice were subcutaneously administered loperamide dissolved in saline (Otsuka Pharmaceutical Co., Ltd., Tokyo, Japan) at a dose of 0.3 or $1 \mathrm{mg} / \mathrm{kg}$.

\section{Experimental Colitis Mouse Model}

Mice were administered 2\% (w/v) DSS (36-50 kDa; MP Biomedicals, Santa Ana, CA, USA) in drinking water ad libitum for 7 days. The control group received only water for 7 days. Cages were cleaned and mice were monitored every day. The DAI was determined as follows: weight loss percentage $(0=$ weight gain, $1=$ $0-5 \%, 2=5-10 \%$, and $3=>10 \%)$, stool consistency $(0=$ normal, $1=$ soft but formed, 2 = very soft, and 3 = diarrhea), and bleeding ( 0 = none, 1 = small spots of blood in stool, 2 = large spots of blood in stool, and 3 = gross bleeding). Mice were euthanized after measuring colonic motility in vivo, and tissue samples were collected for histological analysis.

\section{Determination of Colonic Transit}

A bead expulsion assay was performed to measure colonic transit, as described in previous reports $[8,9]$. Briefly, mice were placed in a mouse holder for $5 \mathrm{~min}$ to induce defecation before testing. Saline or loperamide was subcutaneously administered $30 \mathrm{~min}$ before bead insertion. Glass beads (1.5 mm diameter; CellPoint Scientific Inc., Gaithersburg, MD, USA) were inserted into the distal colon $(3 \mathrm{~cm}$ past the anus) under isoflurane anesthesia. After inserting the bead, the mice were placed in individual cages. The time between when the mice woke up and when the beads were expelled was measured.

\section{Ultrasonography}

Echocardiographic speckle tracking-based strain imaging was evaluated by a digital microultrasound system (Vevo 3100; FUJIFILM VisualSonics Inc., Toronto, Canada) with a 25- to 55-MHz linear array transducer (MS550S; FUJIFILM VisualSonics Inc.) [18, 19]. The transducer and heated imaging platform were integrated into an imaging station with a vital monitoring system (Vevo Imaging Station; FUJIFILM VisualSonics Inc.) to hold the mice in position and support the scanning with the manipulation techniques. All mice were kept under anesthesia with $1.5-2.0 \%$ isoflurane gas in air at a flow rate of $1.0 \mathrm{~L} / \mathrm{min}$ during the entire scanning procedure. The animals were placed in a supine position on a heated imaging platform to maintain constant body temperature. Body fur was removed from the abdomen using a commercially available depilatory cream.

$\mathrm{B}$-mode imaging was performed in a transverse orientation for a 700-frame cine loop with a 70 frames per second sampling rate. Acquired cine loop datasets were imported into speckle tracking software (VevoStrain; FUJIFILM VisualSonics Inc.). In this study, 
we applied echocardiographic speckle tracking-based strain imaging to analyze proximal colonic motility in mice. Cine loop datasets were selected from 3 to 4 consecutive cardiac cycles, and a trace line was placed on the boundary between the proximal wall of the colon and the inner cavity to analyze colonic wall displacement and strain rate. We defined locomotion activity of the colonic wall as the average radial displacement and strain rate between any number of speckle points on the trace line for all time frames. Among the trace lines set on the intestinal wall, we focused on 20 points set on the serous side of the intestinal wall and calculated the migration distance of the inner points and the strain rate between the 2 paired inner and outer points. The displacement, which is the actual distance that the colonic wall boundary moved, was calculated by averaging the maximum distance per unit of time of each point tracked in B-mode for all points. The strain rate, which is the rate of relative change in colonic wall length per unit time, was calculated by converting the maximum rate of change $(\Delta L)$ calculated from the difference between the minimum length $\left(L_{0}\right)$ and maximum length $(L)$ between the 2 points to a unit of time.

Functional blood vessel visualization and quantification in the colon were performed using the power Doppler mode. The percent vascularity, which is an index of relative vascular density, was calculated as the ratio of color-coded voxels to total voxels in the region of interest.

\section{Hematoxylin and Eosin Staining}

Colon tissue samples were fixed with $10 \%$ neutral buffered formalin for $24 \mathrm{~h}$, embedded in paraffin, and cut into $6-\mu \mathrm{m}$-thick sections for staining. Sections were stained with hematoxylin and eosin and imaged with an optical microscope (ACT-10; Nikon, Tokyo, Japan).

\section{Whole-Mount Colonic Muscularis Preparation}

Immunohistochemical analyses were performed using wholemount colonic muscularis preparations. Whole-mount colonic muscularis samples were prepared as previously reported [20]. The samples were cut open, and the muscularis was gently dissected under a stereomicroscope. The muscularis tissue sheet pinned to the silicon cover was permeabilized with ice-cold acetone for $10 \mathrm{~min}$. After fixation, tissue samples were washed with Tris-buffered saline.

\section{Immunofluorescence}

Immunohistochemical staining was performed to assess macrophage infiltration, as described in previous reports [20]. Briefly, fixed whole-mount preparations were incubated with 1:500 diluted rat anti-CD68 antibody (Bio-Rad Laboratories, Inc., Hercules, CA, USA) and rabbit anti-PGP9.5 antibody (UltraClone Limited, Isle of Wight, UK). After labeling with Alexa Fluor 488 or 594 secondary antibody (Life Technologies, Gaithersburg, MD, USA), the samples were observed under a laser scanning confocal microscope (EZ-C1; Nikon). The average number of CD68-positive cells was calculated using images of 3 randomly selected areas in each sample.

\section{Statistics}

The numerical data were expressed as the mean \pm standard error of the mean. Statistical differences were analyzed by an unpaired Student's $t$ test for comparison between 2 groups and by 1-way ANOVA, followed by Tukey's test or Dunnett's test for comparisons between more than 2 groups. Statistical significance was defined as $<0.05$.

Ultrasonography of Colonic Motility

\section{Results}

Ultrasonography Enables the Quantification of Changes in Colonic Motility in Loperamide-Treated Mice

To determine the inhibitory effects of loperamide on colonic motility, we first measured colonic transit by a bead expulsion assay. When loperamide was subcutaneously administered at a concentration of 0.3 or $1.0 \mathrm{mg} / \mathrm{kg}$, the bead expulsion time was significantly prolonged compared to that when mice were treated with saline (Fig. 1a). Based on these results, we explored the establishment of a new method for quantifying colonic motility using loperamide-treated mice.

We next examined whether ultrasonography could quantify murine colonic motility. In this study, we used an ultrasonic imaging system for small animals (Fig. 1b). Simple ultrasonography in B-mode detected the structure of the murine colon. Before the administration of loperamide (Pre), the colon exhibited active peristalsis in B-mode (online suppl. Video 1; for all online suppl. material, see www.karger.com/doi/10.1159/000511851). In contrast, after the administration of loperamide at a concentration of $0.3 \mathrm{mg} / \mathrm{kg}$, murine colon peristalsis was markedly suppressed. Additionally, loperamide at a concentration of $1.0 \mathrm{mg} / \mathrm{kg}$ more potently suppressed colonic peristalsis in mice than loperamide at a concentration of $0.3 \mathrm{mg} / \mathrm{kg}$. This suppression of colonic peristalsis was very prominent at 30 and $60 \mathrm{~min}$ after the administration of loperamide (online suppl. Video 2). These results indicated that ultrasonography could detect changes in colonic motility caused by loperamide. In subsequent experiments, we examined a quantitative method to evaluate colonic motility to enable more objective assessments. Colon locomotion activity was defined by the average displacement and strain rate, which were quantified by analyzing the change in speckle points on trace lines drawn on long-axis ultrasound images (Fig. 1c). The movement of speckle points on the colonic wall decreased 15 min after the administration of loperamide at a concentration of 0.3 or $1.0 \mathrm{mg} / \mathrm{kg}$. Additionally, at 30 and $60 \mathrm{~min}$ after the administration of loperamide, the movement of speckle points significantly decreased (Fig. 1d). Colon locomotion activity, as calculated from the change in speckle points, began to decrease at $15 \mathrm{~min}$ and decreased markedly at 30 and 60 min after the administration of loperamide (Fig. 1e). Taken together, these results indicate that ultrasonography can rapidly detect and quantify changes in colonic motility.

Digestion 2021;102:731-741 

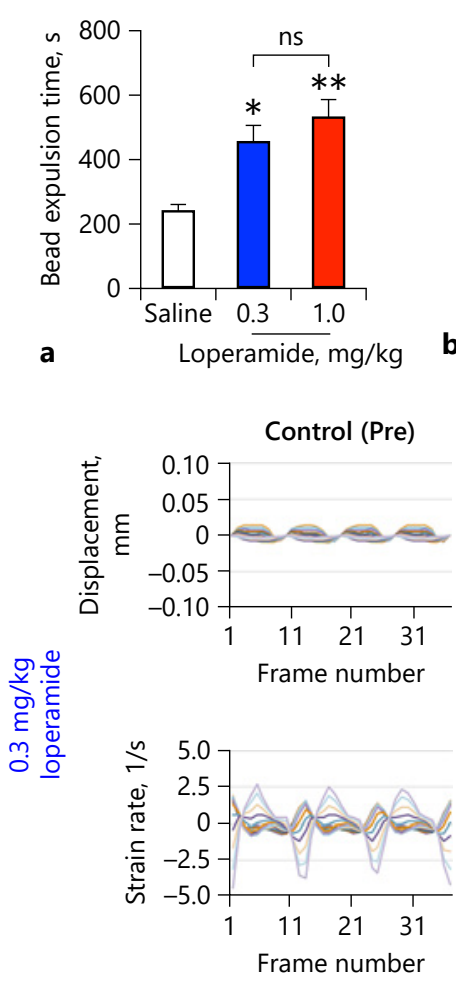

Control (Pre)

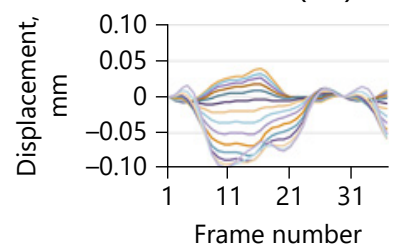

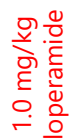

d
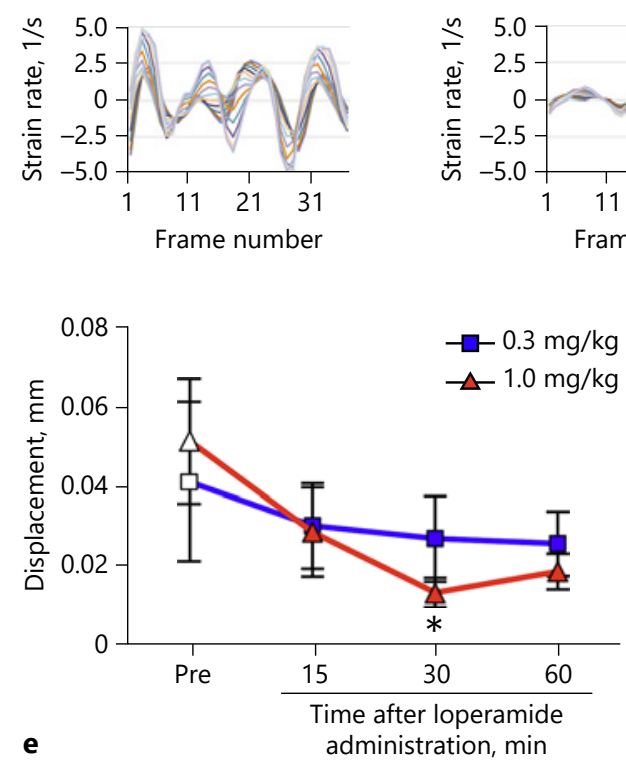

Drawing a trace line : green
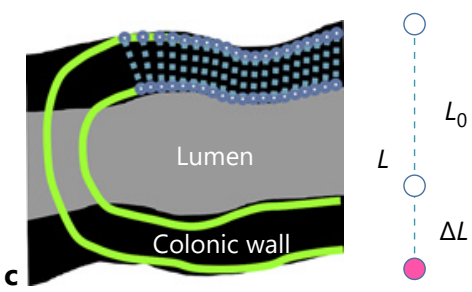

Displacement, $\mathrm{mm}=\Delta L$

Strain rate, $1 / s=\frac{\Delta L / L_{0}}{\Delta t}$
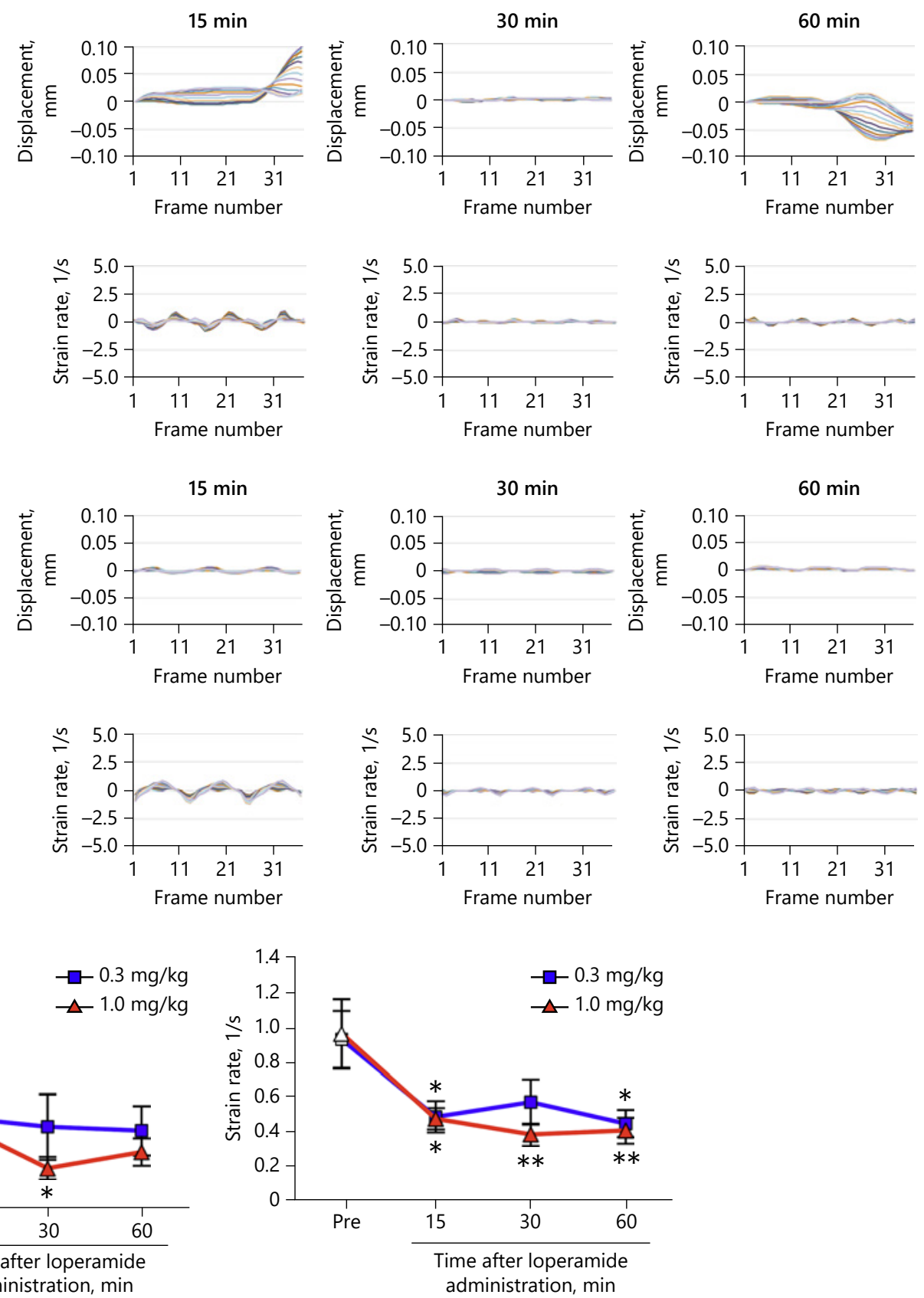

(For legend see next page.) 


\section{DSS Induces Severe Colitis and Decreases Colonic}

Motility in Mice

To further investigate the usefulness of ultrasonography in assessing colonic motility, we used a colitis mouse model as a pathological model. Recent studies have shown that inflammation in the GI tract causes disturbances in GI motility [18, 19]. Based on this finding, we first induced colitis by administering DSS to mice. The administration of DSS gradually reduced the body weight of mice (online suppl. Fig. 1a). The disease activity index based on body weight and fecal status significantly increased in DSS-treated mice (Fig. 2a; online suppl. Fig. 1b). After necropsy, spleen weight, which was correlated with the degree of inflammation, significantly increased in DSS-treated mice (online suppl. Fig. 1c, d). The colon of DSS-treated mice was significantly shorter than that of control mice (online suppl. Fig. 1e, f). Next, histological analysis was performed to examine the level of inflammation in the colon. DSS-treated mice showed severe enteritis with the destruction of the epithelium, abnormal crypts, and infiltration of neutrophils compared to control-treated mice (Fig. 2b). Additionally, immunostaining for CD68 was performed to assess macrophage infiltration in the myenteric plexus layer. PGP9.5 was used as a marker protein for enteric neurons to identify the myenteric plexus layer. In control tissues, only resident macrophages were observed in the myenteric plexus layer. On the other hand, a large number of macrophages were observed in the tissues of DSS-treated mice (Fig. 2c, d). These characteristic findings suggest that the administration of DSS causes severe colitis with diarrhea and gross bleeding. Finally, to assess the colonic motility of the colitis model mice, the colonic transport ability was examined using a bead expulsion assay. The bead expulsion time in colitis model mice was significantly longer than that in control mice (Fig. 2e). These results indicate that the DSS-induced colitis mouse model was characterized by a marked de-

Fig. 1. Quantification of changes in colonic motility by real-time ultrasonography. a The bead expulsion time in saline- or loperamidetreated mice. Each column shows the mean \pm SEM $(n=5-6)$. b The diagnostic ultrasound imaging systems used in this study. All mice were kept under anesthesia with $2 \%$ isoflurane in air during the entire scanning procedure. c Representative images of the long axes of the colon with trace lines (green) drawn for the quantification of colonic motility. A trace line was placed on the boundary between the proximal wall of the colon and the inner cavity to analyze colonic wall displacement and strain rate. These parameters were calculated by converting the maximum rate of change $(\Delta L)$ calculated from the crease in colonic motility with severe inflammation. Based on these results, we decided to validate the usefulness of ultrasonography using DSS-induced colitis mouse models in subsequent experiments.

\section{Ultrasonography Quantitatively Detects Colonic Motility Disorders in DSS-Induced Colitis Mouse Models}

We applied a method to quantify colonic motility using ultrasonography and examined whether it could detect a decrease in colonic motility in colitis model mice. Simple B-mode ultrasonography showed a large amount of fecal retention, suggesting a decrease in colonic motility in colitis model mice (Fig. 3a). In control mice, the colon exhibited active peristalsis in B mode (online suppl. Video 3), whereas in colitis model mice, colonic peristalsis was significantly suppressed (online suppl. Video 4). Consistent with these imaging findings, the movement of speckle points on the colonic wall was significantly reduced in colitis model mice (Fig. 3b). The average displacement and strain rate calculated from the change in speckle points were significantly reduced in colitis model mice (Fig. 3c). These results suggest that ultrasonography can quantitatively detect a decrease in colonic motility caused by colitis.

\section{Ultrasonography Noninvasively Detects the Presence \\ of Colonic Inflammation in Mice}

Color Doppler ultrasound that is optimized to detect blood flow can identify hypervascularity, which suggests active inflammation. Inflammation in the GI tract disrupts normal GI motility. We hypothesized that the detection of inflammation in the GI tract by ultrasonography could support the assessment of GI motility. Simple B-mode ultrasonography exhibited thickening and irregularities in the colonic wall in colitis model mice compared to control mice (Fig. 4a). Next, blood flow in the colonic wall was quantified by color Doppler ultrasound.

difference between the minimum length $\left(L_{0}\right)$ and maximum length $(L)$ between the 2 points to a unit of time. $\mathbf{d}$ Representative images of the change in speckle points on the trace lines in control mice (Pre) and at 15,30 , and $60 \mathrm{~min}$ after the administration of loperamide $(0.3$ or $1 \mathrm{mg} / \mathrm{kg}$ ). Original B-mode images are shown in online suppl. Videos 1 and 2. e Quantification of locomotion activity calculated from (d). Locomotion activity of the colonic wall was defined as the average radial displacement and strain rate between any number of speckle points on the trace line for all time frames. Each column shows the mean \pm SEM $(n=11) .{ }^{*} p<0.05,{ }^{* *} p<0.01$; significantly different from control mice (Pre). SEM, standard error of the mean. 

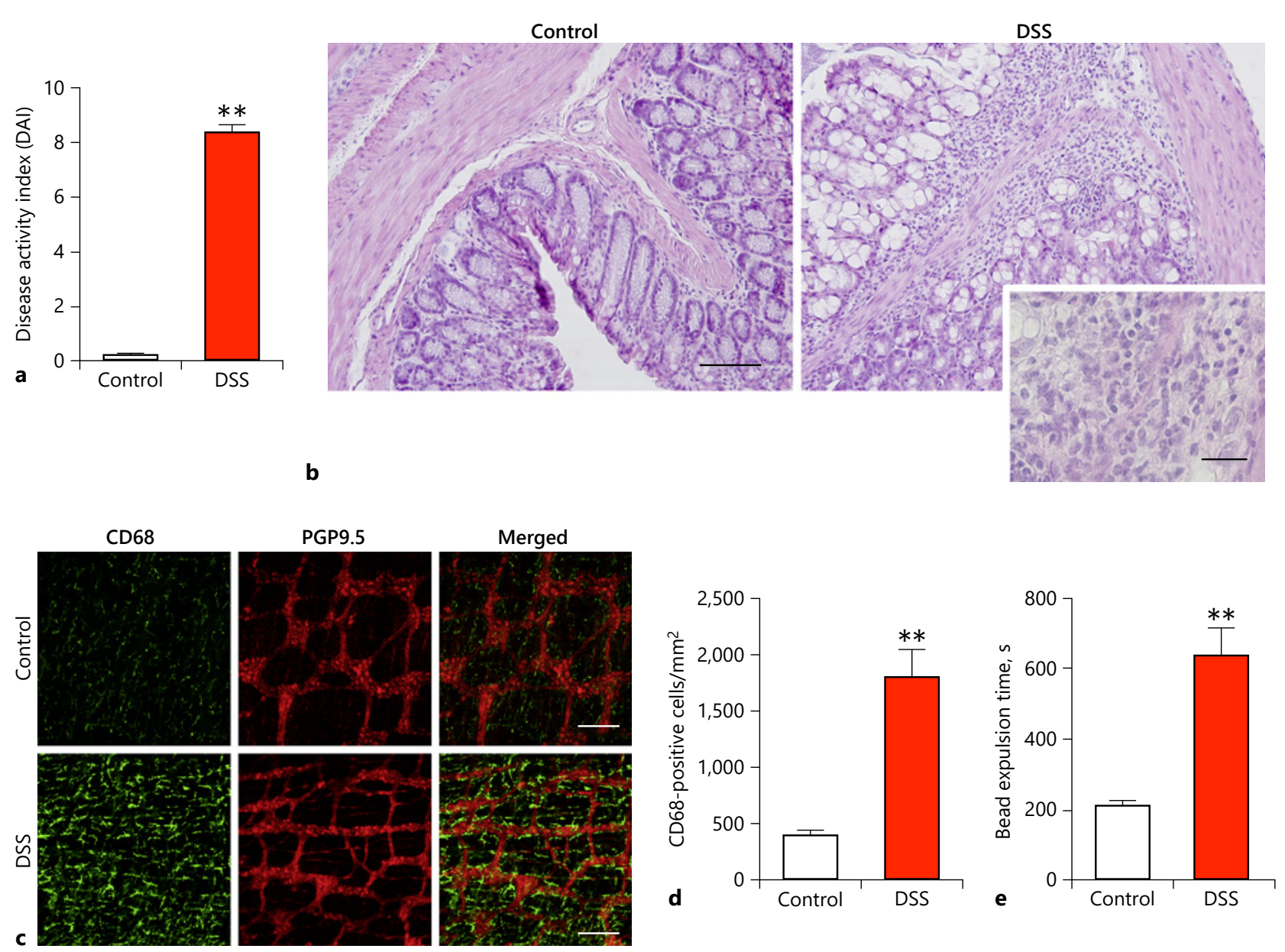

Fig. 2. Characterization of DSS-induced colitis mouse models. a DAI after the administration of DSS in mice. Mice were administered $2 \%(\mathrm{w} / \mathrm{v})$ DSS in drinking water ad libitum for 7 days. The control group received only water for 7 days. Each column shows the mean \pm SEM $(n=7-9)$. $\mathbf{b}$ Hematoxylin and eosin-stained colon sections in DSS-induced colitis mice. Scale bar, $100 \mu \mathrm{m}$. Inset: Neutrophil infiltration. Scale bar, $25 \mu \mathrm{m}$. c Representative results of immunostaining for CD68 (a macrophage marker, green) and PGP9.5 (a neuron marker, red) in whole-mount preparations of

In control mice, no excessive hypervascularity was detected (Fig. 4b, left). In contrast, the findings suggested that excessive vascularity was observed in colitis model mice (Fig. 4b, right). The quantification of blood flow in color images revealed significantly increased blood flow in the colonic wall in colitis model mice (Fig. 4c). These results suggest that ultrasonography is a noninvasive method for quantitatively detecting inflammation in the murine colon. colonic muscularis obtained from control and DSS-induced colitis mice. Scale bar, $100 \mu \mathrm{m}$. d Quantification of the number of macrophages per $1 \mathrm{~mm}^{2}$ of the myenteric plexus layer. Each column shows the mean $\pm \operatorname{SEM}(n=5-6)$. e The bead expulsion time in DSS-induced colitis mice. Beads were inserted into the distal colon $(3 \mathrm{~cm}$ past the anus) under isoflurane anesthesia. Each column shows the mean $\pm \operatorname{SEM}(n=5-7) .{ }^{* *} p<0.01$; significantly different from the control. DSS, dextran sodium sulfate; DAI, disease activity index; SEM, standard error of the mean.

\section{Discussion}

In this study, we established a novel in vivo method for the noninvasive quantification of murine colonic motility using ultrasonography. The quantitative method using ultrasonography was able to quantitatively detect a decrease in colonic motility caused by loperamide or colitis. Additionally, ultrasonography was able to noninvasively assess inflammatory pathology by rapidly detecting intes- 

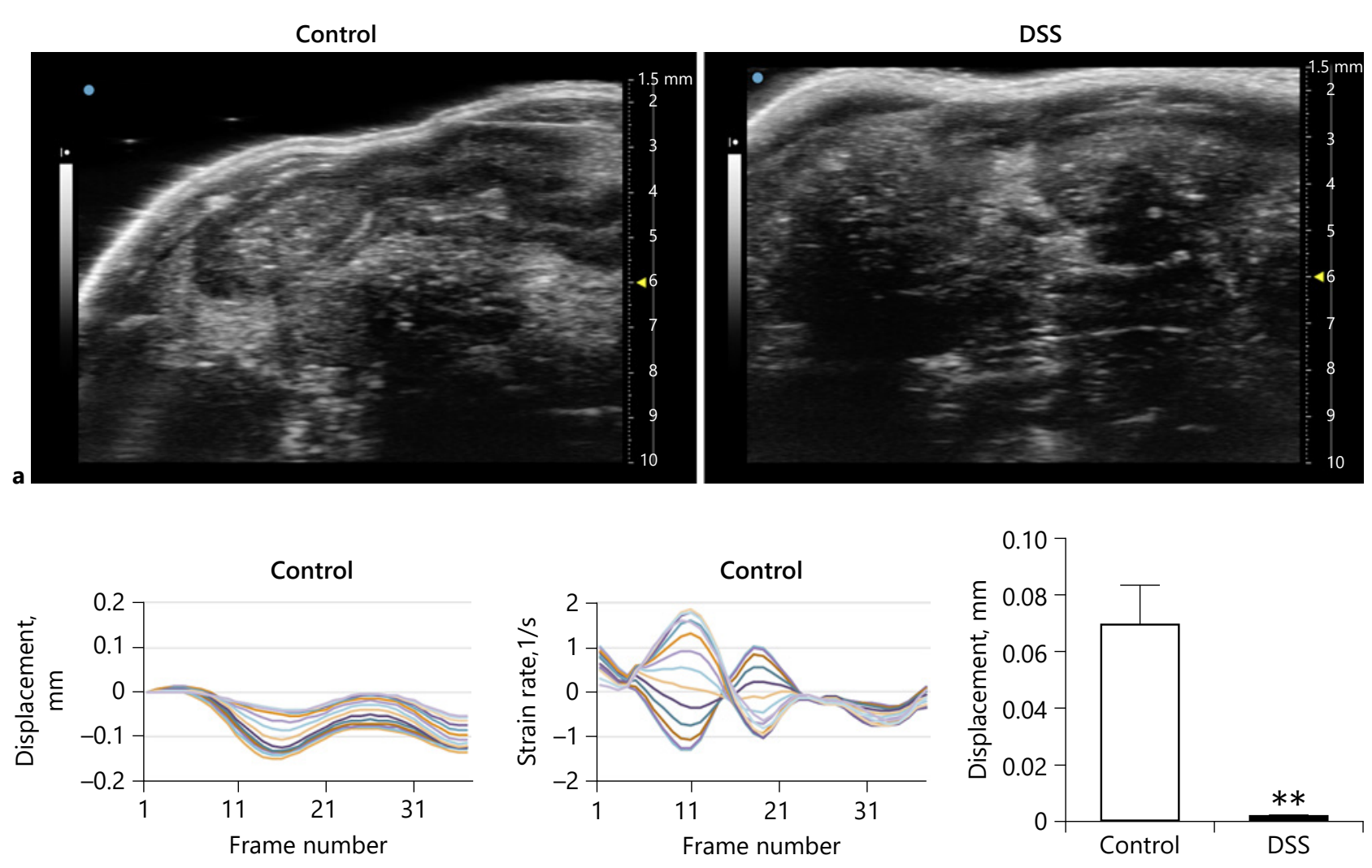

DSS
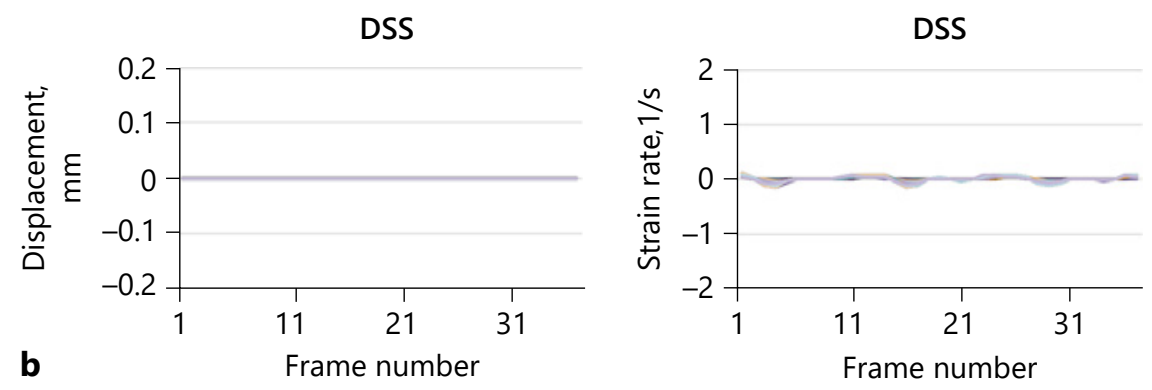

Fig. 3. Ultrasonography objectively detects decreased colonic motility in colitis mice. a Representative ultrasonographic images of a longitudinal cross-section of the murine colon. The left side of the image (probe orientation) shows the proximal side of the colon. These images reveal the presence of large amounts of feces in the colon in mice with colitis, suggesting a disturbance in colonic motility. b Representative images of the change in speckle points on the trace lines in control or colitis mice. Original B-mode im-

tinal thickening and hypervascularity in colitis mice. This study showed that ultrasonography could objectively monitor the change in murine colonic motility over time. The advantage of ultrasonography is that analysis combining morphological evaluation with imaging can be ages are shown in online suppl. Videos 3 and 4. c Quantification of locomotion activity calculated from (b). Locomotion activity of the colonic wall was defined as the average radial displacement and strain rate between any number of speckle points on the trace line for all time frames. Each column shows the mean $\pm \operatorname{SEM}(n=8-9)$. ${ }^{* *} p<0.01$; significantly different from control mice. SEM, standard error of the mean. 


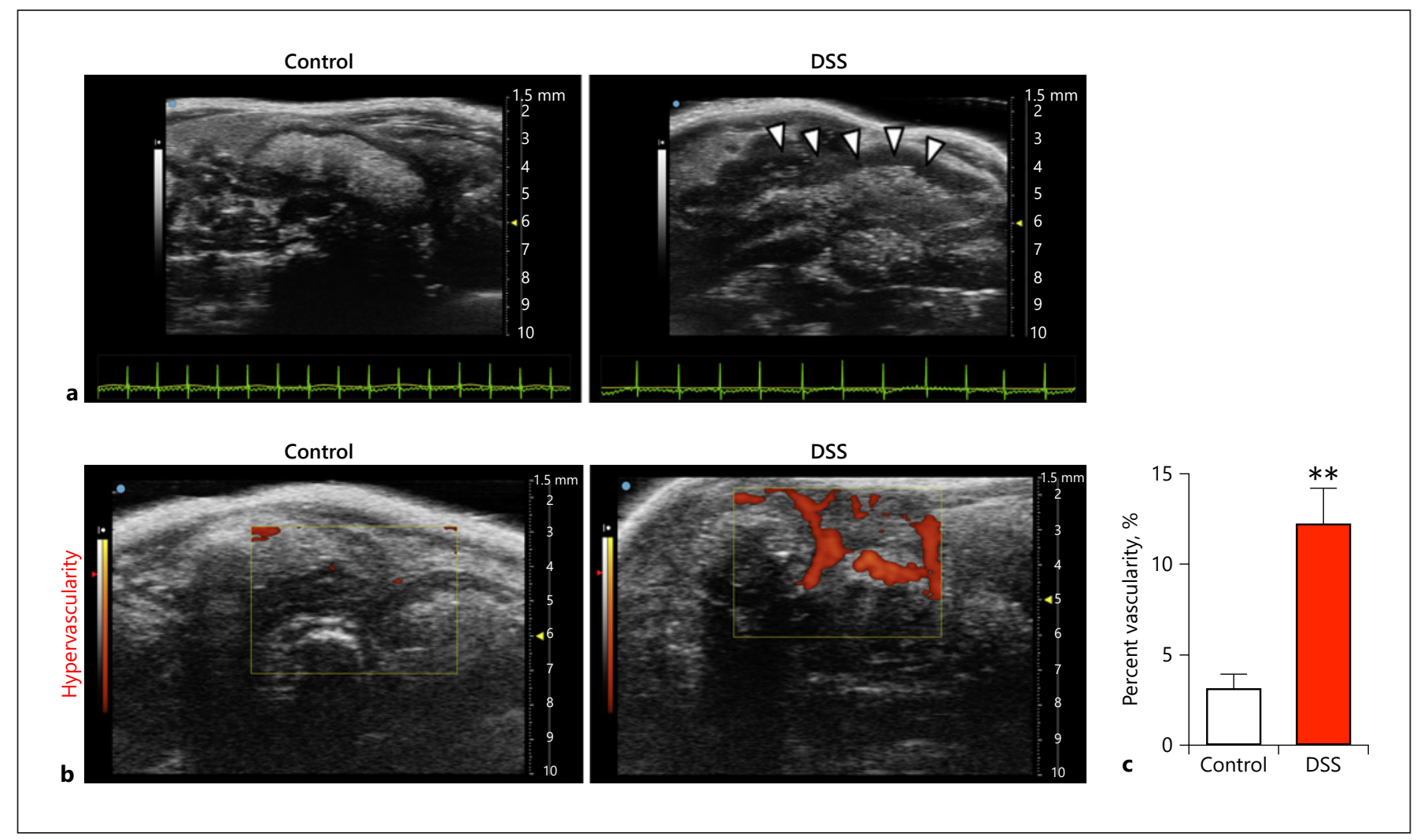

Fig. 4. Noninvasive detection of colonic inflammation using ultrasonography in mice. a Representative ultrasonographic images of a longitudinal cross-section of the murine colon. The left side of the image (probe orientation) shows the proximal side of the colon. These images show changes in the colonic lumen in control or colitis mice. White triangles indicate wall thickening and the loss of normal layer structure in the colon. $\mathbf{b}$ Representative ultrasonographic images of active inflammation in the colon characterized by increased hyperemia on color Doppler ultrasound. c Quantification of the percent vascularity calculated from (b). The percent vascularity, which is an index of relative vascular density, was calculated as the ratio of color-coded voxels to total voxels in the region of interest. Each column shows the mean \pm SEM $(n=5)$. ${ }^{* *} p<0.01$; significantly different from the control. SEM, standard error of the mean.
A bead expulsion assay is used to assess murine colonic motility in vivo $[8,9]$. Previous studies have shown that this method can cause stress-induced defecation due to the insertion of glass beads, which can have a significant effect on measurements [9]. Therefore, there is a need to develop a simpler and noninvasive measurement technique to assess colonic motility. In the present study, we demonstrated that ultrasound imaging could detect the suppression of colonic peristalsis in response to treatment with loperamide; however, the assessment based on diagnostic imaging greatly depends on the knowledge and experience of researchers, and there is a risk of human error $[16,17]$. Thus, it is difficult draw conclusions on the effects of drugs and diseases on colonic motility by subjective assessments. In this study, we examined the locomotion activity of the colonic wall to quantify colonic motility as a solution to this problem. The locomotion activity quantified by ultrasonography was significantly reduced after the administration of loperamide, which was consistent with the ultrasonographic finding of hypoperistalsis. Additionally, the quantitative results were consistent with the bead expulsion assay results, suggesting that ultrasonography accurately reflects colonic motor function. The quantitative analysis method using ultrasonography measures only the locomotion activity of the intestinal wall. Further investigations will be necessary to show that there is a strong correlation between this value and colonic motility. Nevertheless, ultrasonography can obtain quantitative supplemental information on colonic motor function and objectively detect changes in colonic motility. 
In recent years, many reports have proposed several in vivo methods to assess colonic motility, such as wireless motility capsule testing [21-24], radiopaque markers [23, $25,26]$, and scintigraphy $[23,27,28]$ in humans. However, these methods require the oral uptake of special samples and long-term observations and have many limitations when conducting rodent-based tests. Therefore, it is difficult to simply examine the effect of a drug on colonic motility using these methods over time. Additionally, a bead expulsion assay, which is mainly used to assess the colonic transport ability in rodent studies, also requires the observation of animals over long time periods [9]. On the other hand, in this study, ultrasonography revealed that the locomotion activity of the colonic wall began to decrease $15 \mathrm{~min}$ after the administration of loperamide and significantly decreased after 30 and $60 \mathrm{~min}$. Ultrasonography is unique in that it allows the effect of a drug on GI motility to be easily assessed in real time. By taking advantage of this feature, ultrasonography allows measurements at different time points in the same animal. Additionally, we previously reported that ultrasonography can quantitatively assess small intestinal motility in the longitudinal direction over time [29]. The present study shows the usefulness of a new analytical method to quantify changes in colonic motility. These findings suggest that ultrasonography can quickly detect changes in GI motility and provide objective data. The novel in vivo method using ultrasonography could be a research technique that can analyze changes in GI motility as a result of drugs in real time.

Severe murine colitis induced with DSS is known to be most similar to ulcerative colitis (UC) in humans [30]. UC is a type of IBD and is related to the pathophysiology of GI motility disorders. Many reports have demonstrated that suppression of colonic motility is caused in model mice of UC and Crohn's disease [31-34]. In addition, previous reports have shown that in DSS-induced colitis mice, impaired colonic motility is due in part to dysfunction of interstitial cells of Cajal, which are responsible for spontaneous GI contraction [31, 32]. This colonic dysmotility exacerbates colonic transit. In fact, in this study, bead expulsion time in colitis model mice was significantly prolonged. These findings suggest that colonic motility is significantly reduced in DSS-induced colitis model mice. Ultrasonography provided imaging findings showing a large amount of fecal retention in colitis model mice. Consistent with these results, ultrasonography quantitatively detected colonic motility disorders in colitis model mice. Taken together, these findings suggest that ultrasonography is an in vivo method that can objectively detect

Ultrasonography of Colonic Motility the suppression of colonic motility caused by inflammation.

Ultrasonography has been used to diagnose UC in humans and detect intestinal thickening, the loss of normal layer structure, and increased blood flow in the intestinal wall [35]. Thus, ultrasonography can provide images of morphological abnormalities in the intestinal tract. In fact, simple ultrasonography in B-mode detected thickening and irregularities in the colonic wall of colitis model mice. In human clinical practice, color Doppler ultrasound is used to identify increased blood flow, which is indicative of inflammation $[10,35]$. In this study, we performed color Doppler ultrasound in mice and showed that ultrasonography quantitatively detected increased blood flow in the intestinal wall during inflammation. These results suggest that ultrasonography is a useful research tool to noninvasively determine whether inflammation is present in the murine GI tract. A major advantage of ultrasonography is that morphological evaluations are possible in addition to dynamic evaluations of GI motility. The assessment of inflammation in the GI tract using ultrasonography could help detect possible abnormal GI motility.

The novel in vivo method that we established in this study provides an objective assessment, which is essential for investigating the effects of drugs and diseases on colonic motility in basic medical research. The application of ultrasonography, a diagnostic imaging method, enables the dynamic evaluation of murine colonic motility in real time. This is a major feature of ultrasonography that is not available in other methods. In the future, the dynamic evaluation of colonic motility could be important in clinical practice. Currently, the diagnosis of many lower GI tract diseases is subjective and based on clinical markers such as stool morphology and the frequency of defecation rather than the results of physiological measurements of GI motility [36]. However, previous reports have shown that there is only a moderate correlation between stool morphology and colon transit time in humans with constipation [37]. Additionally, the frequency of defecation hardly correlates with changes in colonic motility, and 1 report revealed that this clinical marker is insufficient as an index that reflects colonic motility [38]. These findings suggest that clinical markers do not reliably reflect changes in colonic motility. Therefore, physiological measurements are needed to diagnose abnormal colonic motility, and dynamic assessments using ultrasonography could optimize treatment options in lower GI tract disease. Future work, including validation in dogs and hu- 
mans, will further clarify the potential of this application in human and veterinary clinical practice. On the other hand, ultrasonography has some limitations. One is that this method needs to be performed under anesthesia. Frequent general anesthesia can potentially affect GI motility. Second, ultrasonography is affected by intestinal gas, which makes it difficult to obtain clear images. To obtain a stable and clear image, it is necessary to develop a systematic scanning method and landmark for each animal species to specify an observation site. Although some limitations remain, the new method that we established can quantitatively measure colonic motility and could be an effective in vivo method that contributes to the development of new drugs and research on GI diseases.

In summary, we have presented the first evidence that ultrasonography can be used for the quantitative assessment of colonic motility. Ultrasonography simultaneously provides dynamic and morphological evaluations of colonic motility in real time and allows for quick and objective examinations of colonic motor function. Our findings provide new insights into applications of ultrasonography in the analysis of colonic motor function.

\section{Statement of Ethics}

All procedures were approved by the Institutional Review Board of the University of Tokyo (approval code P18-131). The experimental procedures using mice complied with the Guide for Animal Use and Care published by the University of Tokyo.

\section{Conflict of Interest Statement}

The authors have no conflicts interest to declare.

\section{Funding Sources}

This work was supported by a Grant-in-Aid for JSPS Fellows (20J11240 to K.K.) and Scientific Research from The Ministry of Education, Culture, Sports, Science and Technology (24248050 to M.H., and 18K14604 to N.K.).

\section{Author Contributions}

K.K. and M.H. designed the study. K.K. and Y.T. performed most of the experiments and analyzed the data. K.K. and N.K. wrote the paper. All of the authors reviewed and revised the manuscript.

\section{References}

1 Stanghellini V, Chan FK, Hasler WL, Malagelada JR, Suzuki H, Tack J, et al. Gastroduodenal disorders. Gastroenterology. 2016 May; 150(6):1380-92.

2 Mearin F, Lacy BE, Chang L, Chey WD, Lembo AJ, Simren M, et al. Bowel disorders. Gastroenterology. $2016 \mathrm{Feb} ; 150(6): 1393-407$.

3 Whitehead K, Cortes Y, Eirmann L. Gastrointestinal dysmotility disorders in critically ill dogs and cats. J Vet Emerg Crit Care. 2016 Mar-Apr;26(2):234-53.

4 Vittal H, Farrugia G, Gomez G, Pasricha PJ. Mechanisms of disease: the pathological basis of gastroparesis: a review of experimental and clinical studies. Nat Clin Pract Gastroenterol Hepatol. 2007 Jun;4(6):336-46.

5 Longstreth GF, Thompson WG, Chey WD, Houghton LA, Mearin F, Spiller RC. Functional bowel disorders. Gastroenterology. 2006 Apr;130(5):1480-91.

6 Barnes EL, Kappelman MD, Long MD, Evon DM, Martin CF, Sandler RS. A novel patientreported outcome-based evaluation (PROBE) of quality of life in patients with inflammatory bowel disease. Am J Gastroenterol. 2019 Apr;114(4):640-7.

7 Danese S, Sans M, Fiocchi C. Inflammatory bowel disease: the role of environmental factors. Autoimmun Rev. 2004 Jul;3(5):394-400.

8 Koslo RJ, Burks TF, Porreca F. Centrally administered bombesin affects gastrointestinal transit and colonic bead expulsion through supraspinal mechanisms. J Pharmacol Exp Ther. 1986 Jul;238(1):62-7.

9 Camilleri M, Linden DR. Measurement of gastrointestinal and colonic motor functions in humans and animals. Cell Mol Gastroenterol Hepatol. 2016 Jul;2(4):412-28.

10 Mallouhi A, Bonatti H, Peer S, Lugger P, Con$\operatorname{rad} \mathrm{F}$, Bodner G. Detection and characterization of perianal inflammatory disease: accuracy of transperineal combined gray scale and color Doppler sonography. J Ultrasound Med. 2004 Jan;23(1):19-27.

11 Kusunoki H, Haruma K, Hata J, Kamada T, Ishii M, Yamashita N, et al. Efficacy of mosapride citrate in proximal gastric accommodation and gastrointestinal motility in healthy volunteers: a double-blind placebo-controlled ultrasonographic study. J Gastroenterol. 2010 Dec;45(12):1228-34.

12 Stevens JE, Gilja OH, Gentilcore D, Hausken T, Horowitz M, Jones KL. Measurement of gastric emptying of a high-nutrient liquid by $3 \mathrm{D}$ ultrasonography in diabetic gastroparesis. Neurogastroenterol Motil. 2011 Mar;23(3): 220-4.

13 Tsukamoto A, Ohno K, Tsukagoshi T, Maeda S, Nakashima K, Fukushima K, et al. Realtime ultrasonographic evaluation of canine gastric motility in the postprandial state. J Vet Med Sci. 2011 Sep;73(9):1133-8.
14 McLellan J, Wyse CA, Dickie A, Preston T, Yam PS. Comparison of the carbon 13-labeled octanoic acid breath test and ultrasonography for assessment of gastric emptying of a semisolid meal in dogs. Am J Vet Res. 2004 Nov;65(11):1557-62.

15 Welsh C, Jarrin J, Daneman A, Belik J. In vivo ultrasound assessment of gastric emptying in newborn mice. J Pediatr Gastroenterol Nutr. 2015 Mar;60(3):322-6.

16 Asthana AK, Friedman AB, Maconi G, Maaser C, Kucharzik T, Watanabe M, et al. Failure of gastroenterologists to apply intestinal ultrasound in inflammatory bowel disease in the Asia-Pacific: a need for action. J Gastroenterol Hepatol. 2015 Mar;30(3):446-52.

17 Panes J, Bouhnik Y, Reinisch W, Stoker J, Taylor SA, Baumgart DC, et al. Imaging techniques for assessment of inflammatory bowel disease: joint ECCO and ESGAR evidencebased consensus guidelines. J Crohns Colitis. 2013 Aug;7(7):556-85.

18 Bauer M, Cheng S, Jain M, Ngoy S, Theodoropoulos C, Trujillo A, et al. Echocardiographic speckle-tracking based strain imaging for rapid cardiovascular phenotyping in mice. Circ Res. 2011 Apr;108(8):908-16.

19 An X, Wang J, Li H, Lu Z, Bai Y, Xiao H, et al. Speckle tracking based strain analysis is sensitive for early detection of pathological cardiac hypertrophy. PLoS One. 2016 Feb;11(2):e0149155. 
20 Kaji N, Nakayama S, Horiguchi K, Iino S, Ozaki H, Hori M. Disruption of the pacemaker activity of interstitial cells of Cajal via nitric oxide contributes to postoperative ileus. Neurogastroenterol Motil. 2018 Mar;30(8): e13334.

21 Saad RJ, Hasler WL. A technical review and clinical assessment of the wireless motility capsule. Gastroenterol Hepatol. 2011 Dec; 7(12):795-804.

22 Camilleri M, Thorne NK, Ringel Y, Hasler WL, Kuo B, Esfandyari T, et al. Wireless pHmotility capsule for colonic transit: prospective comparison with radiopaque markers in chronic constipation. Neurogastroenterol Motil. 2010 Aug;22(8):874-e233.

23 Rao SS, Camilleri M, Hasler WL, Maurer AH, Parkman HP, Saad R, et al. Evaluation of gastrointestinal transit in clinical practice: position paper of the American and European neurogastroenterology and motility societies. Neurogastroenterol Motil. 2011 Jan;23(1):823.

24 Rao SS, Kuo B, McCallum RW, Chey WD, DiBaise JK, Hasler WL, et al. Investigation of colonic and whole-gut transit with wireless motility capsule and radiopaque markers in constipation. Clin Gastroenterol Hepatol. 2009 May;7(5):537-44.

25 Rao SS. Constipation: evaluation and treatment of colonic and anorectal motility disorders. Gastroenterol Clin North Am. 2007 Sep; 36(3):687-x.

26 Metcalf AM, Phillips SF, Zinsmeister AR, MacCarty RL, Beart RW, Wolff BG. Simplified assessment of segmental colonic transit. Gastroenterology. 1987 Jan;92(1):40-7.
27 Deiteren A, Camilleri M, Bharucha AE, Burton D, McKinzie S, Rao AS, et al. Performance characteristics of scintigraphic colon transit measurement in health and irritable bowel syndrome and relationship to bowel functions. Neurogastroenterol Motil. 2010 Apr; 22(4):415-e95.

28 Keller J, Bassotti G, Clarke J, Dinning P, Fox $\mathrm{M}$, Grover M, et al. Expert consensus document: advances in the diagnosis and classification of gastric and intestinal motility disorders. Nat Rev Gastroenterol Hepatol. 2018 May;15(5):291-308.

29 Kishi K, Kaji N, Endo M, Tsuru Y, Oikawa T, Hori M. Development of a quantitative method for evaluating small intestinal motility using ultrasonography in mice. Exp Anim. 2019 Aug;68(3):381-9.

30 Okayasu I, Hatakeyama S, Yamada M, Ohkusa T, Inagaki Y, Nakaya R. A novel method in the induction of reliable experimental acute and chronic ulcerative colitis in mice. Gastroenterology. 1990 Mar;98(3):694-702.

31 Lu C, Lu H, Huang X, Liu S, Zang J, Li Y, et al. Colonic transit disorder mediated by downregulation of interstitial cells of Cajal/anoctamin-1 in dextran sodium sulfate-induced colitis mice. J Neurogastroenterol Motil. 2019 Apr;25(2):316-31.

32 Dai YC, Zheng L, Zhang YL, Chen X, Chen DL, Wang LJ, et al. Jianpi Qingchang decoction regulates intestinal motility of dextran sulfate sodium-induced colitis through reducing autophagy of interstitial cells of Cajal. World J Gastroenterol. 2017 Jul;23(26):472434.
33 Kiyosue M, Fujisawa M, Kinoshita K, Hori M, Ozaki H. Different susceptibilities of spontaneous rhythmicity and myogenic contractility to intestinal muscularis inflammation in the hapten-induced colitis. Neurogastroenterol Motil. 2006 Nov;18(11):1019-30.

34 Sato K, Ohkura S, Kitahara Y, Ohama T, Hori $\mathrm{M}$, Sato $\mathrm{M}$, et al. Involvement of CPI-17 downregulation in the dysmotility of the colon from dextran sodium sulphate-induced experimental colitis in a mouse model. Neurogastroenterol Motil. 2007 Jun;19(6):50414.

35 Bryant RV, Friedman AB, Wright EK, Taylor KM, Begun J, Maconi G, et al. Gastrointestinal ultrasound in inflammatory bowel disease: an underused resource with potential paradigm-changing application. Gut. 2018 May;67(5):973-85.

36 Fox MR, Kahrilas PJ, Roman S, Gyawali CP, Scott SM, Rao SS, et al. Clinical measurement of gastrointestinal motility and function: who, when and which test? Nat Rev Gastroenterol Hepatol. 2018 Sep;15(9):568-79.

37 Saad RJ, Rao SS, Koch KL, Kuo B, Parkman HP, McCallum RW, et al. Do stool form and frequency correlate with whole-gut and colonic transit? Results from a multicenter study in constipated individuals and healthy controls. Am J Gastroenterol. 2010 Feb;105(2): 403-11.

38 Russo M, Martinelli M, Sciorio E, Botta C, Miele E, Vallone G, et al. Stool consistency, but not frequency, correlates with total gastrointestinal transit time in children. J Pediatr. 2013 Jun;162(6):1188-92. 